\title{
Irkutsk Incoherent Scatter Radar: history, present and future
}

\author{
Andrey V. Medvedev ${ }^{1}$ and Alexander P. Potekhin ${ }^{1, \dagger}$ \\ ${ }^{1}$ Institute of Solar-Terrestrial Physics of the Siberian Branch of the Russian Academy of \\ Sciences, Irkutsk, 664033, Russia \\ $\dagger$ deceased \\ Correspondence: Andrey V. Medvedev (medvedev@iszf.irk.ru)
}

Received: 21 December 2018 - Revised: 19 June 2019 - Accepted: 2 July 2019 - Published: 21 August 2019

\begin{abstract}
The article focuses on the history of ionospheric research using the incoherent scatter method at the Institute of Solar-Terrestrial Physics and development of the only incoherent scatter radar in Russia, which is located near Irkutsk. It describes the radar features and the current situation of research at the Irkutsk Incoherent Scatter Radar (IISR).

Operating modes and types of measurements of the radar are specified. There is a brief description of the original measurement techniques that were developed considering the IISR features such as the frequency principle of scanning and receiving of one linear polarization of a scattered signal. The main feature of the IISR is the possibility of obtaining absolute values of the ionospheric plasma electron density. The automatic method for constructing the electron density vertical profile is based on registration of vertical profiles of a rotation phase of the polarization plane of a scattered signal. The method does not require calibration with additional facilities.
\end{abstract}

\section{Historical background}

In the Soviet Union, the 1960s was a period of growth in all branches of science and, above all, in physics. At that time, special attention was paid to programs of space exploration and research of the environment and technical activities, new for mankind. In 1961, the Siberian Institute of Earth Magnetism, Ionosphere and Radiowave Propagation (SibIZMIR) was established in Irkutsk (since 1992, the Institute of SolarTerrestrial Physics). The main objectives of the institute were defined in the area of research of solar activity and its impact on the Earth's magnetosphere and ionosphere. To meet these challenges, a network of observatories from the southern to northern frontiers of eastern Siberia was set up immediately; construction of major research facilities began: a large solar vacuum telescope at the shore of Lake Baikal, a horizontal coronagraph telescope at the border with Mongolia, and a solar radio telescope. At that time, it was assumed that sufficient information on the ionosphere and the magnetosphere of the Earth could be obtained through a wide ground-based network of ionosondes and magnetometers. In 1964, a young physicist named Geliy Zherebtsov (see Fig. 1), a graduate of
Irkutsk University, became the head of one of the institute's divisions, the polar magnetic-ionospheric station near Norilsk.

His studies showed that the existing information was clearly not enough to detect unambiguous links between the events on the Sun and specific scenarios of development of ionospheric and magnetospheric disturbances, especially at high latitudes. In order to understand the full spectrum of ionospheric phenomena, new methods and instruments with a broader range of capabilities were required. And these methods were already available. US scientists had developed a method to study the ionospheric plasma using the phenomenon of radio wave incoherent scattering on ionospheric plasma thermal fluctuations (Dougherty and Farley, 1960; Farley et al., 1961). In America and Europe, incoherent scatter radars were under construction and starting their operation. Works on developing the first incoherent scatter radar inside the USSR began at the Kharkiv Polytechnic Institute (Emelyanov and Zhivolup, 2013). Zherebtsov issued a proposal to the SibIZMIR leaders to construct a radar of the same type in Norilsk. However, this proposal 


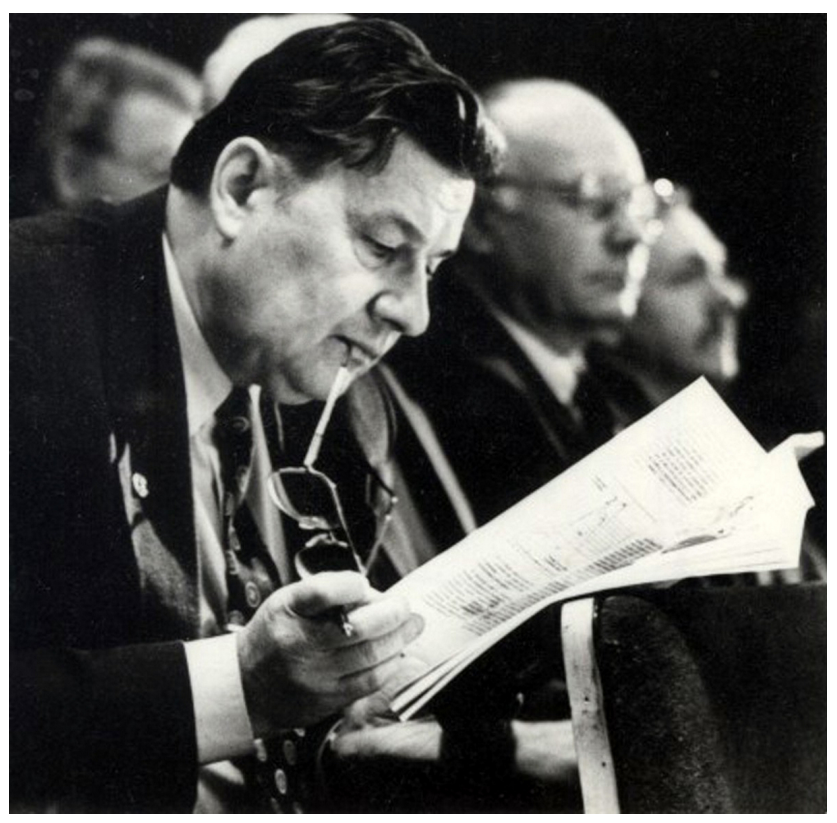

Figure 1. Geliy Alexandrovich Zherebtsov.

faced a rather lukewarm attitude: to construct such an installation in the harsh Polar Circle climate was a far from a simple case. There was significant doubt, but Zherebtsov started preparation work. He talked to representatives of the USSR electronic industry and arranged expeditions to select the future radar location. Works on IS radar construction near Norilsk were interrupted in 1973 when Zherebtsov got his appointment as deputy director of the institute and moved to Irkutsk for the full-time employment. The new job and new wide range of responsibilities put the idea of constructing the IS radar on hold, but Zherebtsov had no doubt: sooner or later, the IS radar for ISTP would be constructed, and this meant that they needed to master a complex technique of processing IS signals and to develop special research equipment for this purpose. By happy fortune it turned out that for experiments in this area, they could possibly use one of the existing military "Dnepr" radar systems (space surveillance and early warning radar system) near Irkutsk (https://en.wikipedia.org/wiki/Dnestr_radar, last access: 13 August 2019). After negotiations, the military gave their permission to use this system for episodic scientific experiments. In 1975, Zherebtsov initiated a special laboratory at the institute, where they designed and constructed the first 40-channel analog spectrograph (see Fig. 2) and developed methods and programs to process data of incoherent scattering.

At this stage, Boris Velichansky, Valery Kulagin, Vladimir Abramov, Alexander Klimov, Alexey Zavorin, and Valery Evstafiev took part in the works. The researchers obtained the first spectra of incoherent scattering (Abramov et al., 1981) (see Fig. 3). On 31 July 1981, they were able to observe the

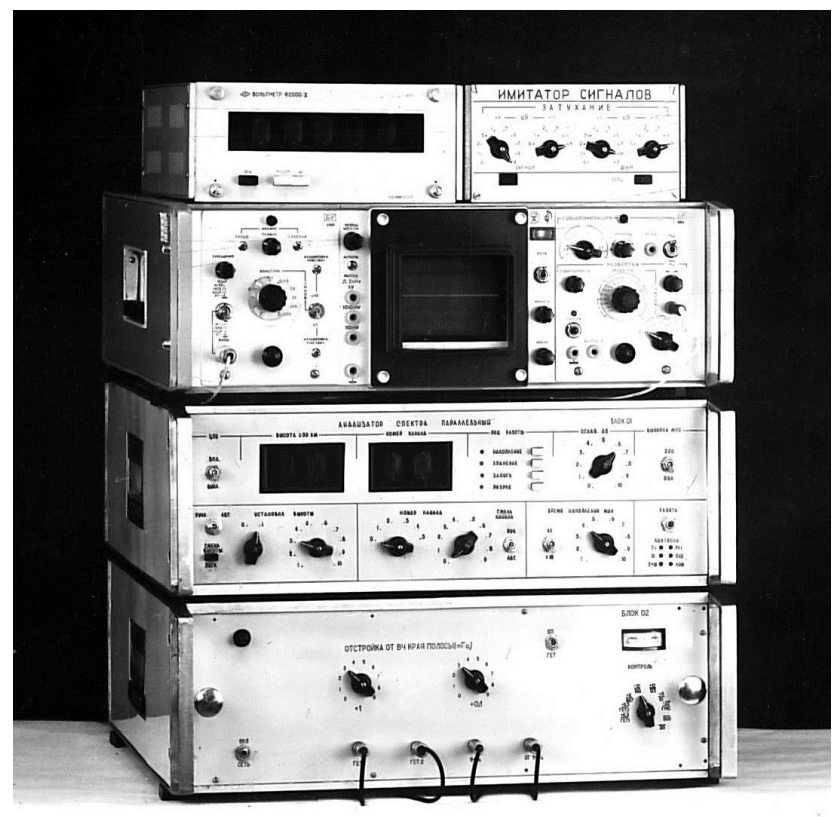

Figure 2. The first 40-channel analog spectrograph.

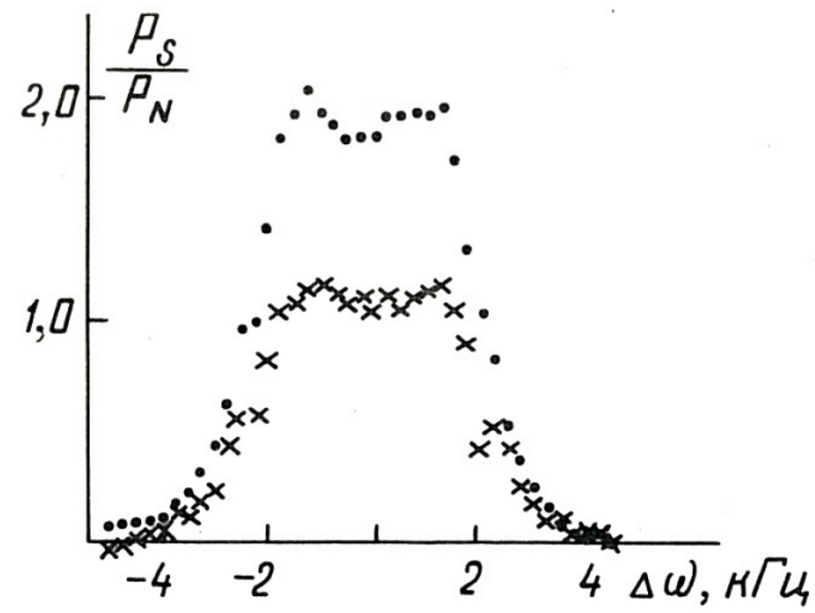

Figure 3. Ratio of incoherent scatter signal power to noise power for heights of $450 \mathrm{~km}$ (dots) and $500 \mathrm{~km}$ (crosses); the horizontal axis shows $\mathrm{kHz}$. The graph of the first spectra is taken from the article published in Russian (Abramov et al., 1981).

ionospheric effects caused by the solar eclipse (Abramov et al., 1982).

It became clear that the Dnepr radars can be used for ionospheric studies, but long-term and regular observations were required to meet the challenges of developing comprehensive physical models of the ionosphere response to solar event impacts. The institute needed its own incoherent scatter radar.

In 1984, Zherebtsov became the director of the institute. The work on constructing IS radar became the first prior- 


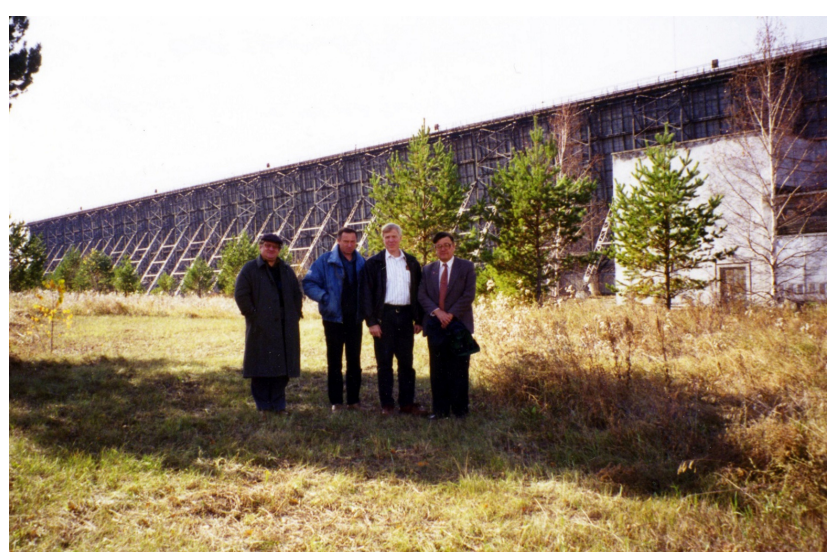

Figure 4. Near the radar antenna. Left to right: Vasily Mikhailovsky, Alexander Potekhin, John Foster, and Gely Zherebtsov.

ity again. But it became possible only in 1993. In the 1990s in Russia, of course, construction of any major scientific facility was out of the question. The structure of the warning radar system was being reduced, and the Ministry of Defence agreed to transfer one of the system radars to the institute instead of deconstructing it. Works commenced to reassign the radar for scientific use. Alexander Potekhin, a researcher specializing in radio physics and ionospheric plasma propagation, headed these works. Igor Orlov, Andrey Medvedev, and Boris Shpynev have joined the works.

At this point, our colleagues from the Millstone Hill observatory, and their leader John Foster who visited the radar in autumn 1997 (see Fig. 4), gave very important support to the future Irkutsk Incoherent Scatter Radar. Foster was able to get a grant from the National Science Foundation for joint research at the IISR and Millstone Hill ISR, and this cooperation was of value for the IISR from both a scientific and financial point of view.

\section{Principal IISR equipment}

The Dnepr radar transferred to the institute is a frequencyscanning monostatic pulse radar. It is located $120 \mathrm{~km}$ northwest of Irkutsk. Figure 5 shows the control room of the Dnepr radar at the time it was transferred to the ISTP in 1993.

The radar potential was determined by the power of its transmitters and the antenna system aperture. The radar twoway antenna had a unique design, which defined its capabilities in many ways. The antenna represented a generating horn with $246 \times 12.2 \mathrm{~m}$ aperture dimensions; lengthwise, it was divided into two symmetrical half-horns, each with its own independent feeder systems and outputs to the receiver (Fig. 6). The antenna gain was $\sim 35 \mathrm{~dB}$. The antenna had a polarization filter; at $30 \mathrm{~dB}$, it suppressed the electric field component transverse to the horn's big axis. In this way, signals of strictly linear polarization were emitted and received.

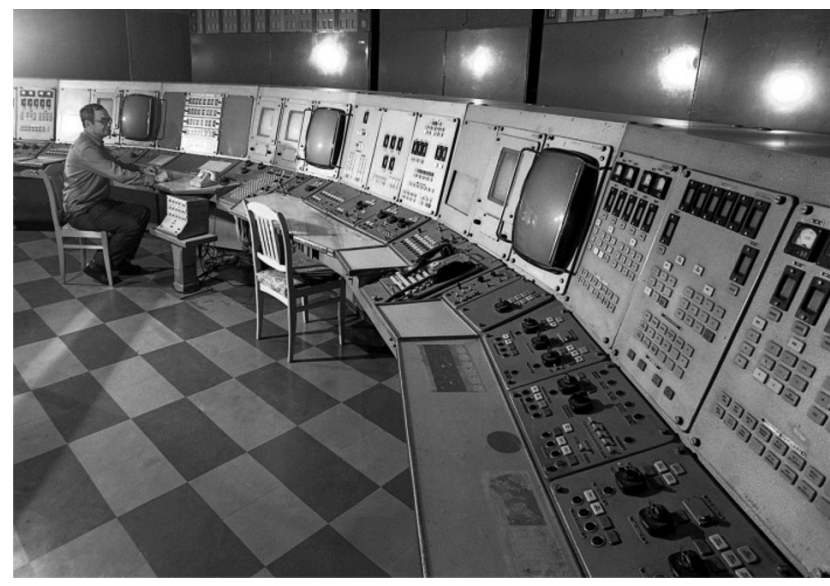

Figure 5. The Dnepr radar control room (1990s).

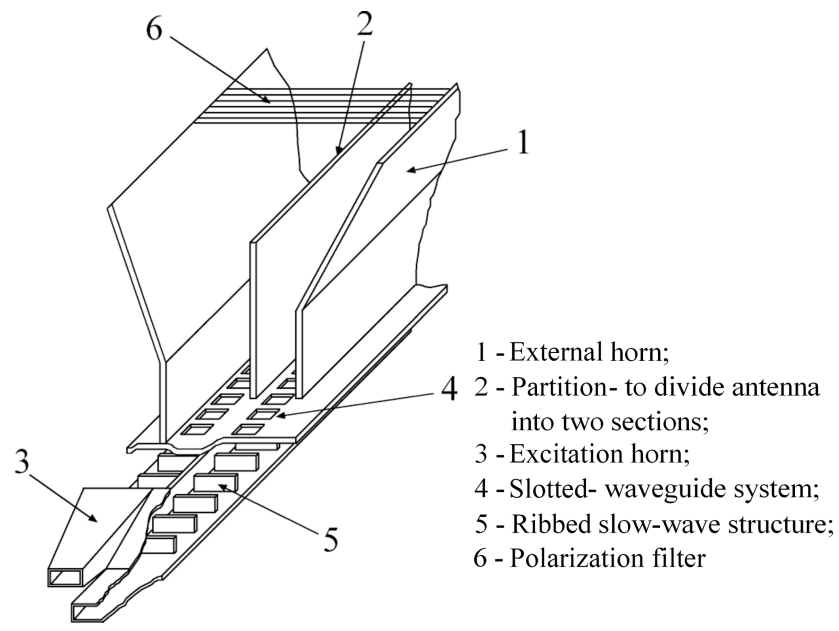

Figure 6. Structure of the IISR antenna.

The extended slotted waveguide excitation system of each of the half-horns ensures the width of the antenna pattern (AP) along the big axis of about $0.5^{\circ}$. The ribbed slow-wave structure changes the wave phase velocity with the operating frequency swaps. As a result, the distribution of initial phases on the emitting slots changes, i.e., the phase front inclination, which ensures the AP scanning in a plane passing through the antenna big axis. At $154 \mathrm{MHz}$ carrier frequency, emission is normal to the horn mouth plane. When the frequency changes in the range of $154-162 \mathrm{MHz}$, it causes the AP inclination of the exciting electromagnetic wave to move up to $30^{\circ}$ away from the antenna aperture normal (Fig. 7). When excitation is fed to the antenna half-horn alternately from the opposite ends of the waveguide, the full angle of scanning is $\pm 30^{\circ}$ relative to the antenna aperture normal. In the rolling plane (along the antenna short axis), the AP of each of the two independent sections of the antenna system is $\sim 20^{\circ}$ wide. The radar cumulative AP is formed by adding the antenna patterns of the two half-horns. During emission, 
the AP shape can be controlled by changing the excitation signal phase difference in the half-horns. In synphased excitation, the AP is $\sim 10^{\circ}$ wide in the rolling plane.

The antenna main axis is $7^{\circ}$ tilted from the north-south direction, and the angle between the antenna aperture normal and zenith is $10^{\circ}$.

The radar has six transmitters, three for each direction of excitation. Two transmitters emit simultaneously (one into each half-horn), and one is in "hot" reserve. The peak power of each transmitter is $1.6 \mathrm{MW}$. The pulse-repetition rate is $24.4 \mathrm{~Hz}$. The duration of a sounding pulse is 70-900 $\mu$ s.

The transmitting equipment was transferred to the institute complete; it has been employed intact until today. But we had to refurbish or newly elaborate all the radar control systems, devices generating the sounding signals, and devices receiving and detecting the scatter signals. Based on opportunities available at that time, it was selected to develop a receiving and detecting unit on the platform of a 30 MFlop response time signal processor and personal computers. In 1995, the first data series of digital registration of incoherent scatter signals with the IISR were obtained.

Technical details of the IISR reconstruction first stage are presented by Zherebtsov et al. (2002).

\section{Second stage of IISR modernization}

The receiving and recording facility that was created at the stage of IISR conversion and development has completed its task. Our scientists worked out algorithms of measuring the ionosphere parameters (Shpynev, 2004), performed longterm measurements of the ionospheric plasma characteristics under various helio-geophysical conditions, including superstorms of solar cycle 23 (Kurkin et al., 2001; Potekhin et al., 2009a), and compared the obtained experimental data with the data by other instruments and known ionospheric models (Foster et al., 1998; Potekhin et al., 2002). For instance, the IISR was intensively used during studies of such a relatively rare phenomenon as mid-latitude coherent echo (radio aurora) - a signal scattered in the E-layer on inhomogeneities extended along the magnetic field lines (Potekhin et al., 1999). Besides, principal shortcomings of the IRNR equipment were revealed, which restricted its diagnostic capabilities. These included the following.

- Insufficient range of reception path linear response $(\sim 40 \mathrm{~dB})$.

- Fast program control of emission and receive modes was not possible.

- Emission of smooth non-modulated pulses only.

- There was no phase coherence of the end-to-end reception-transmission channel.
- Limited capabilities of signal processing devices (no more than six spectra of IS signals per one cycle of sounding).

- Limited capabilities of data registration and storage devices: it was impossible to store sounding information in full.

At the new stage, extension of the IISR diagnostic capabilities became the purpose of fundamental modernization of the entire set of control, receiving and recording devices and signal processing tools, in order to fully use the radar potentials and features of the antenna design.

The following main requirements for the IISR control, receiving and registration systems were formulated.

Multi-channel. During operation, the recording facility is to register and process signals from four receiving channels simultaneously. Changes in the broadband channel should be made to each half-horn of the antenna. This would provide high spatial resolution to measure the electron density profile. Changes in the narrow-band channel are required for spectral and correlation measurements with high-frequency resolution.

Wide dynamic range of the detection system was to exceed the linearity range of the end-to-end reception path (over $70 \mathrm{~dB}$ ) and ensure simultaneous undistorted measurement of power signals from space objects, reflection from local objects (clutter) and coherent echo (radio aurora) against the background of weak incoherent scatter signals.

Detecting and storing the signal full shape should be continuous in the entire range of altitudes studied independently for all reception channels. Signal bands were to be provided in broadband channels at the intermediate frequency of the reception path $300-1250 \mathrm{kHz}$ and in narrow-band channels at the intermediate frequency of $50-250 \mathrm{kHz}$.

Control of the AP shape, fast scanning modes, interferometric measurements - all these should be capable of coordinated fast tuning of the sounding and reception frequencies; they should have a system of transmitter automatic phasing, registration of received signals separately in the two antenna half-horns, and end-to-end coherence of the emissionreception path.

Complex signals with ample opportunities to change the duration of the sounding pulses, types of frequency and phase modulation to increase spatial resolution in observation of ionospheric parameters and while tracking the objects should be generated automatically using software.

The new radar hardware was put into operation in 2005 (Potekhin et al., 2009b).

During this period, the following researchers got involved with the IISR modernization tasks and developing new methods of signal processing: Oleg Berngardt, Viktor Zarudnev, Dmitry Kushnarev, Valentin Lebedev, Sergey Alsatkin, Roman Vasilyev, and Konstantin Grkovich. 


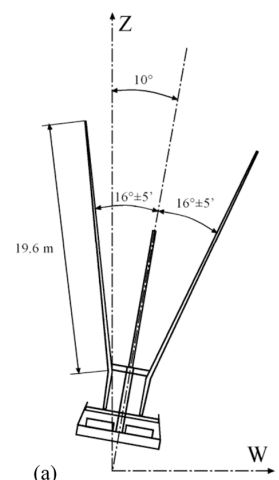

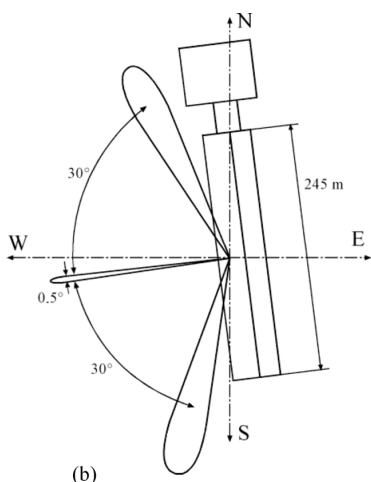

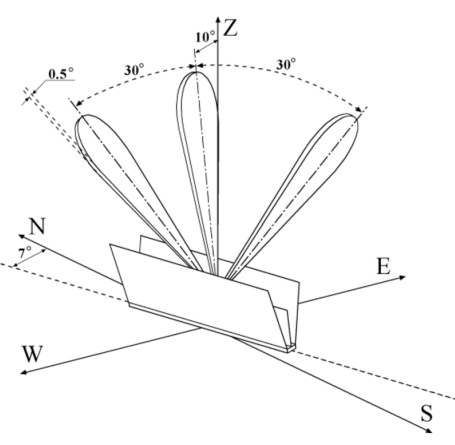

(c)

Figure 7. Dimensions and antenna pattern of the IISR. Antenna dimensions (a, b); AP formation (c).

\section{Operation modes and studies performed}

Modernization of the IISR provided an extended set of operating modes, greater scope and accuracy of data presentation. New functionalities of the radar allowed notable progress in developing new methods for radio diagnostics of the upper atmosphere and near space. The following presents the IISR main features and method description.

\subsection{IISR features when determining characteristics of the ionospheric plasma}

Most of the time, the IISR is operated in ionospheric mode (Potekhin et al., 2008). The principal feature of the Irkutsk radar is the capability to transmit and receive only linear field polarization. When propagating in the ionospheric plasma from the Earth's external magnetic field, electromagnetic waves experience the Faraday rotation. Thus, there occurs a factor of the wave polarization plane dependence on the rotation angle in the radiolocation equation for the Irkutsk radar. This angle, in turn, is determined by integral electron content along the propagation path. At the IISR, this feature is seen as a fading in the vertical power profile of received signals. On the one hand, this factor creates a considerable complication in solving the reverse task of determining plasma temperatures, but on the other hand, it can be used to determine absolute values of electron density on the entire vertical profile (Shpynev, 2004). In this task, the critical element, which determines consistency of the electron density profile restoration method from sounding data, is the procedure of deconvolution of the IR signal power profile with the sounding signal. The procedure is ambiguous, and its stability under highnoise conditions is not always sufficient. With respect to the IISR, this procedure can be avoided if the altitude resolution is better than $5 \mathrm{~km}$. This task was accomplished when new equipment was brought in at the Irkutsk radar. This equipment can generate signals with the required type of modulation, detect the entire sounding information, and conduct suspended processing of signals using software tools. Mathematical modelling of the ionospheric reaction with realistic parameters to coded signals of different shapes (Alsatkin et al., 2009) made it possible to identify the main types of signals that provide the best possible accuracy in recovery of the density profile in different geophysical conditions, and to develop methods to automatically select the type of probing signal and processing method. This work resulted in an automated process of measuring the vertical electron density profiles in real time, which worked steadily in the range of $N m F 2$ values $2 \times 10^{5}-2 \times 10^{6} \mathrm{~cm}^{-3}$. Figure 8 demonstrates an example for the period of 12-15 January 2013. Figure 8a shows power profiles of IS signals measured with an optimal code sequence of $150-200 \mu$ s total duration after coordinated processing. Note that there is a distinct separation of maxima and minima that do not require additional deconvolution with the sounding signal. Figure $8 \mathrm{~b}$ shows electron density profiles related to these measurements. The profiles were obtained using the automated procedure. Figure $8 \mathrm{c}$ shows time variability of electron density at the heights of $250 \mathrm{~km}$ (blue line) and $325 \mathrm{~km}$ (red line), which approximately correspond to the heights of the day and night ionization maxima.

The processing of long data series for vertical electron density profiles in different seasons and at various levels of solar activity revealed some interesting peculiarities in the electron content behavior above the altitude of the ionization maximum (Zherebtsov et al., 2017).

Another IISR feature distinguishing it from similar radars and adding further difficulties to the signal processing procedure is the frequency principle of scanning. The IISR emission direction depends on the carrier frequency. When horizontal ionization gradients occur in the plane of scan, this fact can cause distortion of the IS signal spectrum and make it asymmetrical. In this case, the $\Delta E$ asymmetry parameter is related to the horizontal gradient of the electron density $\partial N_{\mathrm{e}} / \partial x$ along the scanning axis with $\partial N_{\mathrm{e}} / \partial x=\Delta E$. $N_{\mathrm{e}} /(2 \Delta F R \xi)$, where $\Delta F$ is the IS spectrum half-width, $R$ is a slant distance, and $\xi=6.5 \times 10^{-5} \mathrm{rad} \mathrm{kHz}^{-1}$ is the coefficient of the AP inclination angle dependence on frequency (Potekhin et al., 2008). This IISR feature can also lead to a scenario where discrete space radio sources, which pass 

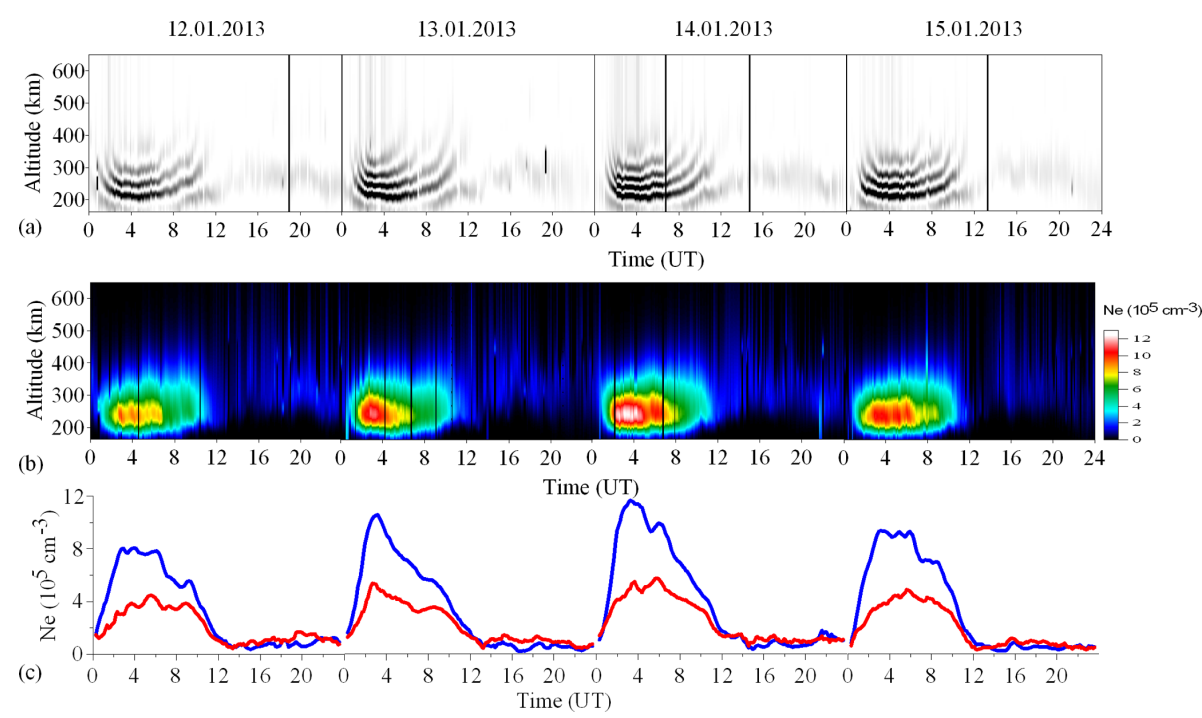

Figure 8. (a) Vertical profiles of IS signal power, measured using the 11-element code sequence with a total duration of $150 \mu$ s. (b) Vertical electron density profiles from the automated procedure. (c) Time variability of the electron density at $259 \mathrm{~km}$ (blue line) and $325 \mathrm{~km}$ (red line).

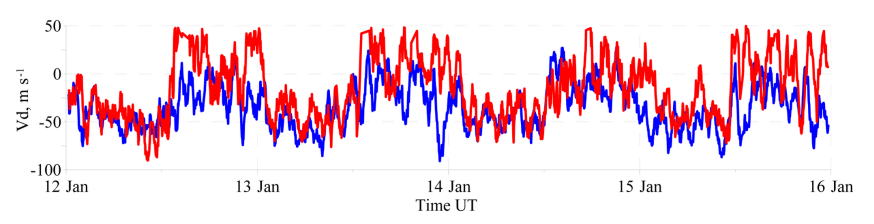

Figure 9. Estimated plasma drift velocity (line of sight) at $250 \mathrm{~km}$ (blue) and $350 \mathrm{~km}$ (red) height.

through the antenna pattern, will also regularly contribute to the spectrum asymmetry (by adding a variable-frequency noise component). In view of this IISR feature, a procedure was developed for analytical separation of the asymmetry coefficient $\Delta \varepsilon$ and Doppler shift of the IS spectrum $\Omega_{\text {dop }}$. Shcherbakov et al. (2009) show that the procedure implies minimizing the functional in the form

$F\left(\Omega_{\mathrm{dop}} \Delta \varepsilon\right)=\sum_{k=1}^{n}\left(\Omega_{\mathrm{dop}} \cdot \tau_{k}+\Delta \varepsilon \cdot \tan \left(\tau_{k}\right)-\psi_{k}\right)^{2}$,

where $\tau_{k}$ is the experimental autocorrelation function (ACF) lag and $\psi_{k}$ is the ACF phase.

Figure 9 presents the derived plasma drift velocity versus time. The blue line shows the plasma drift at $250 \mathrm{~km}$ altitude, and the red line at $350 \mathrm{~km}$.

Based on this method, a technique was developed to calculate the neutral wind meridional component from the IISR data (Shcherbakov et al., 2015).

An important issue in studies of the ionospheric plasma is the use of special methods to increase the spatial resolution without losing spectral resolution. Due to significant frequency dependence of the IISR antenna pattern, the use of standard techniques (alternating codes and pseudo- random codes) can lead to large errors. To solve this problem at IRNR, we developed a new sounding mode - the effective subtraction technique (Berngardt and Kushnarev, 2013). It allows us to increase the spatial resolution by combination of narrow-band radio pulses of different duration. This technique allows estimation of the ionospheric data in two modes: with high spatial and low time resolution, and with low spatial and high time resolution. It is possible to use these modes at the same time without changing the operating mode of the radar, only by using different mathematical data processing.

\subsection{Study of traveling ionospheric disturbances}

Starting from September 2005, after completion of the second modernization phase, regular ionospheric measurements at the IISR have been conducted in two-path mode. This mode involves alternate transmission of sounding pulse sequences at two frequencies corresponding to two directions $15-20^{\circ}$ apart. Given that the IS signal's integration time in each direction is normally $1-10 \mathrm{~min}$, this mode opens the feasibility of getting two independent and simultaneous profiles of the ionospheric plasma parameters. These new functional capabilities of the IISR allow measurement of spatial gradients of the ionospheric parameters; in particular, they were used to develop radio methods of reconstructing the 3D structure of traveling ionospheric disturbances (TIDs) and studying conditions of internal gravity wave propagation in the upper atmosphere. It resulted in a series of publications (Medvedev et al., 2009, 2013, 2015, and 2017). 


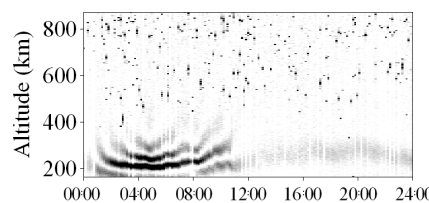

(a)

Time (UT)

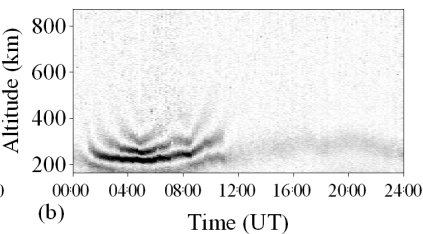

Figure 10. Diurnal variations of the IS signal power profile (a) before SO selection and (b) after SO removal.

\subsection{Observation of space objects with Irkutsk IS radar}

Since 2006, the average operation time in ionospheric radar mode has been about $1500 \mathrm{~h} \mathrm{yr}^{-1}$. Several dozens of space objects (SOs) pass over the IISR antenna AP every hour. On the one hand, the SO is a significant factor of additional noise and distortions during analysis of the space plasma parameters. On the other hand, the observed SOs can be interesting for many fundamental and applied tasks. After modernization, we can now install a special software module at the IISR that can sort out SO signals in real time; see Fig. 10 for an example.

Tens of thousands of SO passes are observed in a month of automated ionospheric measurements with the IISR. Data are processed, and the following characteristics are received: range, line-of-sight velocity, azimuth, location angle, and signal-to-noise ratio (Lebedev et al., 2012). The received data are recorded in a database (http://rp.iszf.irk.ru:8080/iisr/spu/, last access: 13 August 2019). Currently, the database contains information on more than 450000 passes.

Figure 11 presents the SO altitude distribution recorded with the IISR in 2007-2015. To compare with the catalog prediction, we used the SO orbit two-line element data from the Space-Track website (https://www.space-track.org, last access: 13 August 2019). According to the catalog, more than 11000 SOs may pass through the IISR sector. For each of these SOs, we calculated the ephemerid and path characteristics relative to the IISR. As a result, we obtained the altitude distribution of SOs, both observed and calculated from the catalog. These distributions are shown in Fig. 12 for 20132015. The transparent histograms with black borders present distributions of the observed SOs. According to Fig. 12, the number of observed SOs exceeds their calculated number in almost all ranges.

In each series, we have measured the following characteristics: distance $(R)$, line-of-sight velocity $(V)$, azimuth angle $(\varepsilon)$, space object elevation $(\gamma)$, and signal amplitude. When the signal-to-noise ratio is high $(S / N>10)$, the typical root mean square deviations for $R, V, \varepsilon$, and $\gamma$ are $100 \mathrm{~m}$, $10 \mathrm{~m} \mathrm{~s}^{-1}, 5 \mathrm{arcmin}$, and $5 \mathrm{arcmin}$, respectively. This accuracy is sufficient to determine the space object orbit in the next SO passing over the IISR. Two of six Keplerian elements of the orbit can be determined with high accuracy: inclination and right ascension of the $\mathrm{SO}$ ascending node (Lebedev et al., 2012).

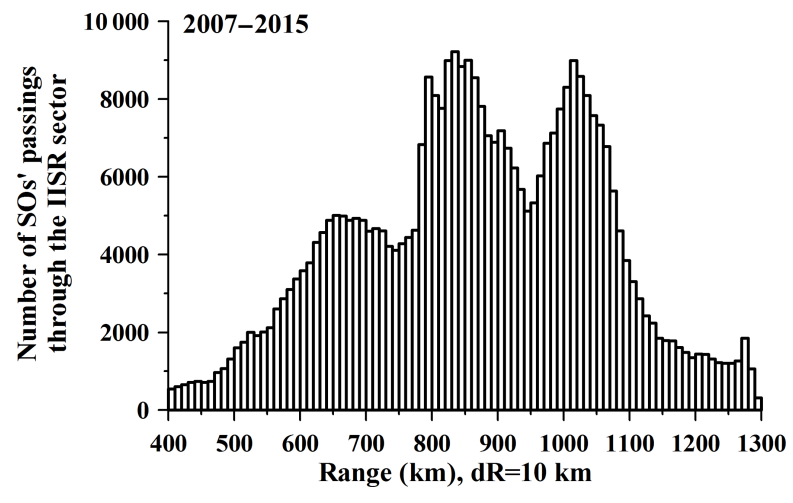

Figure 11. Altitude distributions of space objects in 2007-2015.

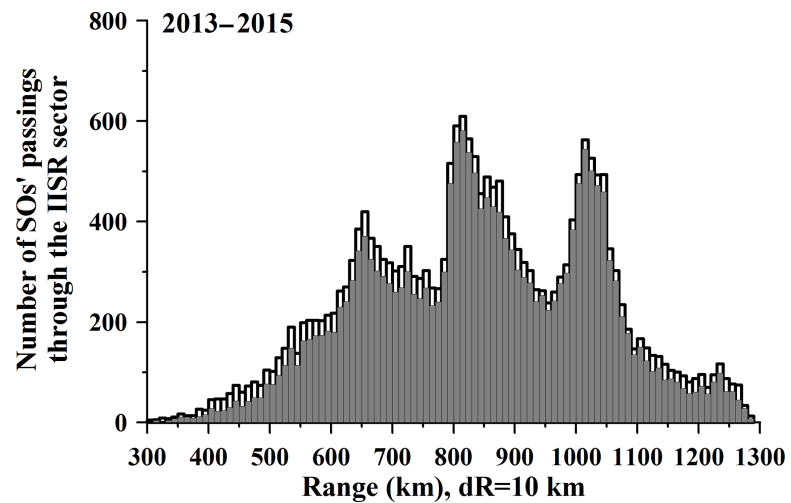

Figure 12. Observed space objects (transparent light gray color); cataloged space objects (dark gray color).

\subsection{Passive observations}

Due to high operation costs, the IS radars only operate a few days a month. The main cost is the use of powerful transmission devices. Utilization of the Irkutsk radar for regular ionospheric studies and special experiments is about $100 \mathrm{~d} \mathrm{yr}^{-1}$. The rest of the time it is possible to use its receiving equipment and antenna system in passive mode and to arrange observations of background radio emission from space. The IISR has been operating in radio astronomical observation mode since May 2011. This implies a regular procedure of signal registration without sending a sounding pulse. In passive mode, the operating frequency is $149-163 \mathrm{MHz}$ and the coverage sector width is approximately $45^{\circ}$. The entire range is split into 88 frequencies spaced at $163 \mathrm{kHz}\left(\sim 0.5^{\circ}\right)$. Thus, taking into account the repetition frequency of $1 / 24 \mathrm{~s}$, scanning of the entire sector takes $3.67 \mathrm{~s}$. IISR control software changes the receiving frequency in such a way as to provide a continuous cycle of scanning in the coverage sector. In this mode, the IISR can be regarded as a multipath panoramic riometer with the 88-lobe antenna pattern extended along the meridian line. This mode determines a complex coherence 


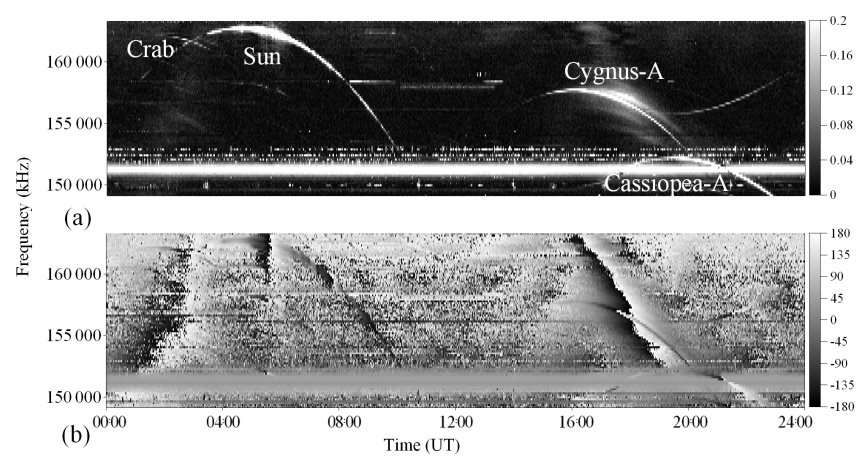

Figure 13. Variation of space noise during a $24 \mathrm{~h}$ period; (a) modulus and (b) phase of the correlation coefficient.

function defined as follows:

$\eta=\frac{\left\langle U_{\mathrm{u}} \cdot U_{\mathrm{d}}^{*}\right\rangle}{\sqrt{\left\langle\left|U_{\mathrm{u}}\right|^{2}\right\rangle\left\langle\left|U_{\mathrm{d}}\right|^{2}\right\rangle}}$,

where $U_{\mathrm{u}, \mathrm{d}}$ are complex amplitudes of signals in upper (eastern) and lower (western) half-horns, respectively. The scanning results are given in Fig. 13.

Passive observation in continuous mode of sky scanning is a new measurement. It allows us to use the IISR as an effective radio astronomical instrument, which makes it possible to study the dynamics and degree of solar radiation spatial inhomogeneity at about $2 \mathrm{~m}$ wavelength and to measure parameters of fine-scale ionospheric irregularities on the basis of registered scintillations of radio signals from discrete space sources (Vasilyev et al., 2017).

\subsection{Special experiments}

Special operation modes are intended to carry out a wide range of radio experiments at the IISR. The new set of IISR instrumentation can provide flexible software control of transmission and receiving modes. Since 2007, the Institute of Solar-Terrestrial Physics, Central Research Institute of Machine Building, and the Russian Rocket Cosmic Corporation "Energy" have been conducting active space experiments, "Radar-Progress" (before 2010, the experiment was called "Plasma-Progress"), aimed at studying the space-time characteristics of ionospheric disturbances arising during operation of liquid-propellant engines onboard the Progress cargo spacecraft.

Orbital manoeuvering subsystem engines of the Progress transport spacecraft (TSC) were used as a source of disturbances for ionospheric and radar signature characteristics. The altitude of the TSC orbit was about $340 \mathrm{~km}$. In order to perform this experiment, the ground-based Radio-Optic Complex of ISTP SB RAS including the IISR has been involved. Each burn started once a day exactly over the radar. The exhaust directions as well as the amounts of injected products changed from flight to flight. During the "PlasmaProgress" experiments, high-precision measurements of TSC coordinate and reflecting characteristics and space-time disturbances of plasma parameters were executed. Multiple radar beams allowed us to simultaneously observe both the modified and unmodified ionosphere. The analysis of the data has shown that burning of even relatively weak TSC engines causes considerable changes in both the electron density profile and the TSC radar cross section (Potekhin et al., 2009c; Khakhinov et al., 2010).

During these active space experiments we used a complex operational mode with transmission of smooth pulses, phase-shift pulses, and pulses with continuous linear frequency modulation. These sequences were transmitted in a variable-frequency grid, which depends on the specific experiment geometry.

\section{Conclusion}

After total modernization, since 2006 to the present time, the IISR has worked over $800 \mathrm{~d}$ in the ionospheric sounding mode. The sounding was carried out in lasting series (normally, a week as minimum) in different seasons and under different geophysical conditions. The ISPT database contains over 3 TB of raw sounding data. Since 2011 till now, in the intervals between active observations, the IISR has been operated in the radio astronomical mode of observations of solar radio emission and registration of signal scintillations from space radio sources. The total time of radio astronomical observations exceeds $700 \mathrm{~d}$.

Total modernization allowed introduction of new original methods of research at the IISR. An automatic method is developed for constructing the electron density vertical profile based on registration of the vertical profile of a rotation phase of the polarization plane of a scattered signal. The method allows reconstruction of absolute values of electron density in real time without calibration by additional facilities. Due to automated measurements, it is possible to carry out studies on the basis of extensive statistics. In particular, we can note a series of studies of travelling ionospheric disturbances (TIDs). Investigation of the TID 3-D structure and full vector of their propagation velocity revealed that over $60 \%$ of TIDs observed in all seasons and times of day correspond to the dispersion ratio typical for internal gravity waves. Resulting from these studies, the method was first offered to detect not only meridional, but also zonal components of the neutral wind velocity in the upper atmosphere.

At present, the IISR is a modern, multifunctional research instrument capable of addressing a wide range of near-Earth space exploration tasks. For example, as part of the Russian Luna-Resurs mission, bistatic radar detection experiments are planned in addition to monostatic radar detection performed in two frequency ranges from the Luna-26 orbiter. It is assumed that the Moon's plasma shell will be studied 
along with its uppermost layer. The objective of this study is to analyze the possibility of bistatic radar detection of the uppermost layer and radio occultation of the Moon's ionosphere using the IISR and RLK-L onboard radar system, developed for the Luna-Resurs orbital module (Yushkova et al., 2018).

The unique capabilities of the radar attract new researchers. In recent years, young scientists Viktor Tashlykov and Artem Setov have asserted themselves with interesting works. For example, Viktor Tashlykov is involved with construction of properly modified ambiguity functions to decrease systematic errors in plasma temperature determination (Tashlykov et al., 2018), and Artem Setov is developing the absolute calibration methods for the IISR (Setov et al., 2018).

IISR experience has provided essential skills of understanding the upper atmosphere and the near-Earth space; we were also able to reveal advantages both of the method itself and of the radar main equipment employed. This experience will be used to construct a new promising ISMST radar (Potekhin et al., 2016), which is now being designed as a part of the major Russian scientific infrastructure project "National Heliogeophysical Complex of the Russian Academy of Science" (http://en.iszf.irk.ru/images/0/01/ Booklet_en.pdf, last access: 13 August 2019). We expect the new IS-MST radar to be put into operation before 2023. An important part of the new radar working program is joint operation and mutual calibration of the two radars for several years.

Author contributions. This article is a joint work of both the authors. All preparations, collecting data, wording, and structuring were performed together.

Competing interests. The authors declare that they have no conflict of interest.

Special issue statement. This article is part of the special issue "The history of ionospheric radars". It is not associated with a conference.

Review statement. This paper was edited by Asgeir Brekke and reviewed by three anonymous referees.

\section{References}

Abramov, V. G., Boitman, O. N., Velichansky, B. N., Zherebtsov, G. A., Klimov, A. N., Klimov, N. N., Kulagin, V. N., and Khaustov, V. V.: Spectral Measurements during incoherent sounding of the ionosphere, Issledovaniya po geomagnetismu, aeronomii i fizike Solntsa (Research on geomagnetism, aeronomy and solar physics), Nauka, Moscow, 55, 113-118, 1981 (in Russian).
Abramov, V. G., Velichansky, B. N., Klimov, A. N., and Kulagin, V. N.: Spectral measurements by incoherent scatter during the solar eclipse of 31 July 1981, Issledovaniya po geomagnetismu, aeronomii i fizike Solntsa (Research on geomagnetism, aeronomy and solar physics), Nauka, Moscow, 59, 112-115, 1982 (in Russian)

Alsatkin, S. S., Medvedev, A. V., and Kushnarev, D. S.: Analyzing the characteristics of phase-shift keyed signals applied to the measurement of an electron concentration profile using the radiophysical model of the ionosphere, Geomagn. Aeronomy, 49, 200-205, https://doi.org/10.1134/s0016793209070305, 2009.

Berngardt, O. I. and Kushnarev, D. S.: Effective subtraction technique at the Irkutsk Incoherent Scatter Radar: Theory and experiment, J. Atmos. Sol.-Terr. Phy., 105-106, 293-298, https://doi.org/10.1016/j.jastp.2013.03.023, 2013.

Dougherty, J. P. and Farley, D. T.: A theory of incoherent scattering of radio waves by a plasma, P. Roy. Soc. Lond. A Mat., 259, 7999, 1960.

Emelyanov, L. Ya. and Zhivolup, T. G.: History of the development of IS radars and founding of the Institute of Ionosphere in Ukraine, Hist. Geo Space. Sci., 4, 7-17, https://doi.org/10.5194/hgss-4-7-2013, 2013.

Farley, D. T., Dougherty, J. P., and Barron, D. W.: A theory of incoherent scattering of radio waves by a plasma II. Scattering in a magnetic feld, in: P. Roy. Soc. Lond. A Mat., 263, 238-258, 1961.

Foster, J. C., Taran, V. I., Zherebtsov, G. A., Potekhin, A. P., and Erickson, P. J.: Longitudinal studies of the sub-auroral ionosphere using the Millstone Hill, Kharkov, and Irkutsk incoherent scatter radars, 32nd COSPAR Scientific Assembly: Abstracts, Nagoya, Japan, 12-19 July 1998, p. 121, 1998.

Khakhinov, V. V., Potekhin, A. P., Lebedev, V. P., Medvedev, A. V., Kushnarev, D. S., Shpynev, B. G., Zarudnev, V. E., Alsatkin, S. S., Ratovskii, K. G., Podlesny, A. V., and Bryn'ko, I. G.: Radio methods of diagnostics of ionospheric disturbances generated by cargo spacecraft "Progress" on-board engines: Algorithms, instruments and results, in: Proceedings of the Russian scientific conference "Probing the Earth's surface with SAR", Ulan-Ude, Russia, 6-10 September, 2010, 555-571, 2010 (in Russian).

Kurkin, V. I., Nosov, V. E., Potekhin, A. P., Smirnov V. F., and Zherebtsov, G. A.: The March 9, 1997 solar eclipse ionospheric effects over Russian Asian region, Adv. Space Res., 27, 14371440, https://doi.org/10.1016/s0273-1177(01)00030-8, 2001.

Lebedev, V. P., Khakhinov, V. V., and Medvedev, A. V.: On results of space debris observation at Irkutsk Radar of Incoherent Scatter in 2007-2010, Solar-Terrestrial Physics, 20, 97-102, 2012 (in Russian).

Medvedev, A. V., Ratovsky, K. G., Tolstikov, M. V., and Kushnarev, D. S.: Method for Studying the Spatial-Temporal Structure of Wave-Like Disturbances in the Ionosphere, Geomagn. Aeronomy, 49, 775-785, https://doi.org/10.1134/s0016793209060115, 2009.

Medvedev, A. V., Ratovsky, K. G., Tolstikov, M. V., Alsatkin, S. S., and Scherbakov, A. A.: Studying of the spatial-temporal structure of wavelike ionospheric disturbances on the base of Irkutsk incoherent scatter radar and Digisonde data, J. Atmos. Sol.-Terr. Phy., 105-106, 350-357, https://doi.org/10.1016/j.jastp.2013.09.001, 2013. 
Medvedev, A. V., Ratovsky, K. G., Tolstikov, M. V., Alsatkin, S. S., and Scherbakov, A. A.: A statistical study of internal gravity wave characteristics using the combined Irkutsk Incoherent Scatter Radar and Digisonde data, J. Atmos. Sol.-Terr. Phy., 132, 13-21, https://doi.org/10.1016/j.jastp.2015.06.012, 2015.

Medvedev, A. V., Ratovsky, K. G., Tolstikov, M. V., Oinats, A. V., Alsatkin S. S., and Zherebtsov, G. A.: Relation of internal gravity wave anisotropy with neutral wind characteristics in the upper atmosphere, J. Geophys. Res.-Space, 122, 7567-7580, https://doi.org/10.1002/2017JA024103, 2017.

Potekhin, A. P., Berngardt, O. I., Kurkin, V. I., Spynev, B. G., and Zherebtsov, G. A.: Observations of abnormally powerful scattering with ISTP IS-radar, in: Proceedings of SPIE/Sixth International Symposium on Atmospheric and Ocean Optics, Tomsk, Russia, 23-26 June 1999, 3983, 328-335, https://doi.org/10.1117/12.370511, 1999.

Potekhin, A. P., Berngardt, O. I., Zavorin, A. V., Shpynev, B. G., and Tashchilin, A. V.: Comparison of data from Irkutsk incoherent scatter radar with international reference ionosphere IRI-95, in: Proceedings of SPIE/Eighth International Symposium on Atmospheric and Ocean Optics: Atmospheric Physics, Irkutsk, Russia, 25-29 June 2001, 4678, 560-566, https://doi.org/10.1117/12.458491, 2002.

Potekhin, A. P., Medvedev, A. V., Zavorin, A. V., Kushnarev, D. S., Lebedev, V. P., and Shpynev, B. G.: Development of Diagnostic Capabilities of the Irkutsk Incoherent Scattering Radar, Cosmic Res., 46, 347-353, https://doi.org/10.1134/s0010952508040102, 2008.

Potekhin, A. P., Zherebtsov, G. A., Kurkin, V. I., Medvedev, A. V., Ratovsky, K. G., and Shpynev, B. G.: Response of the midlatitude ionosphere to extreme geomagnetic storms of the 23rd solar cycle, Geomagn. Aeronomy, 49, 1218-1222, https://doi.org/10.1134/s0016793209080350, 2009a.

Potekhin, A. P., Medvedev, A. V., Zavorin, A. V., Kushnarev, D. S., Lebedev, V. P., Lepetaev, V. V., and Shpynev, B. G.: Recording and control digital systems of the Irkutsk Incoherent Scatter Radar, Geomagn. Aeronomy, 49, 1011-1021, https://doi.org/10.1134/s0016793209070299, 2009 b.

Potekhin, A. P., Khakhinov, V. V., Medvedev, A. V., Kushnarev, D. S., Lebedev, V. P., and Shpynev, B. G.: Active Space Experiments with the use of the Transport Spacecraft "Progress" and Irkutsk IS Radar, in: Proceedings of PIERS, Moscow, Russia, 18-21 August 2009, 223-227, 2009c.

Potekhin, A. P., Setov, A. G., Lebedev, V. P., Medvedev, A. V., and Kushnarev, D. S.: Prospective IS-MST radar. Potential and diagnostic capabilities, Solar-Terrestrial Physics, 2, 3-16, https://doi.org/10.12737/19444, 2016.
Setov, A. G., Globa, M. V., Medvedev, A. V., Vasilyev, R. V., and Kushnarev, D. S.: First results of absolute measurements of solar flux at the Irkutsk Incoherent Scatter Radar (IISR), Solar-Terrestrial Physics, 4, 24-27, https://doi.org/10.12737/stp43201804, 2018.

Shcherbakov, A. A., Medvedev, A. V., and Kushnarev, D. S.: Correlation method for determining the ionospheric plasma drift velocity at the Irkutsk scatter radar, Geomagn. Aeronomy, 49, 206211, 2009.

Shcherbakov, A. A., Medvedev, A. V., Kushnarev, D. S., Tolstikov, M. V., and Alsatkin, S. S.: Calculation of meridional neutral winds in the middle latitudes from the Irkutsk incoherent scatter radar, J. Geophys. Res.-Space, 120, 10851-10863, https://doi.org/10.1002/2015JA021678, 2015.

Shpynev, B. G.: Incoherent scatter Faraday rotation measurement a radar with single linear polarization, Radio Sci., 39, RS3001, https://doi.org/10.1029/2001RS002523, 2004.

Tashlykov, V. P., Medvedev, A. V., and Vasilyev, R. V.: Backscatter signal model for Irkutsk incoherent scatter radar, Solar-Terrestrial Physics, 4, 24-32, https://doi.org/10.12737/szf42201805, 2018.

Vasilyev, R., Globa, M., Kushnarev, D., Medvedev, A., and Ratovsky, K.: Spectral characteristics of ionospheric scintillations of VHF radiosignal near magnetic zenith, J. Atmos. Sol.-Terr. Phy., 160, 48-55, https://doi.org/10.1016/j.jastp.2017.05.016, 2017.

Yushkova, O. V., Gavrik, A. L., Marchuk, V. N., Yushkov, V. V., Smirnov, V. M., Laptev, M. A., Chernyshev, B. V., Dutyshev, I. N., Lebedev, V. P., Medvedev, A. V., and Petrukovich, A. A.: Bistatic Radar Detection in the Luna-Resurs Mission, Solar Syst. Res., 52, 287-300, https://doi.org/10.1134/S0038094618040081, 2018.

Zherebtsov, G. A., Zavorin, A. V., Medvedev, A. V., Nosov, V. E., Potekhin, A. P., and Shpynev, B. G.: The Irkutsk incoherent scattering radar, J. Commun. Technol. El., 47, 1222-1228, 2002.

Zherebtsov, G. A., Ratovsky, K. G., Klimenko, M. V., Klimenko, V. V., Medvedev, A. V., Alsatkin, S. S., Oinats, A. V., and Lukianova, R. Yu.: Diurnal variations of the ionospheric electron density height profiles over Irkutsk: comparison of the incoherent scatter radar measurements, GSM TIP simulations and IRI predictions, Adv. Space Res., 60, 444-451, https://doi.org/10.1016/j.asr.2016.12.008, 2017. 